\title{
Effects of diethylstilbestrol on the proliferation and tyrosinase activity of cultured human melanocytes
}

\author{
JIANBING TANG, QIN LI, BIAO CHENG, CHONG HUANG and KUI CHEN \\ Department of Plastic Surgery, The Key Laboratory of Trauma Treatment and Tissue Repair of Tropical Area, \\ People's Liberation Army, HuaBo BioPharmaceutical Institute of Guangzhou, General Hospital of Guangzhou \\ Military Command, Guangzhou, Guangdong 510010, P.R. China
}

Received March 13, 2015; Accepted April 24, 2015

DOI: $10.3892 /$ br. 2015.472

\begin{abstract}
The aim of the present study was to observe the effects of different exogenous estrogen diethylstilbestrol (DES) concentrations on the human melanocyte proliferation and tyrosinase activity. Skin specimens were obtained following blepharoplasty, and the melanocytes were primary cultured and passaged to the third generation. The melanocytes were seeded in 96-well plates, each well had $5 \times 10^{3}$ cells. The medium was changed after $24 \mathrm{~h}$, and contained $10^{-4}-10^{-8} \mathrm{M}$ DES. After the melanocytes were incubated, the proliferation and tyrosinase activity were detected by the MTT assay and L-DOPA reaction. DES $\left(10^{-8}-10^{-6} \mathrm{M}\right)$ enhanced the proliferation of cultured melanocytes. The intensity was positively correlated with the concentration of drug. DES, $>10^{-5} \mathrm{M}$, inhibited the melanocytes proliferation or even produced the toxicity effect. Following the addition of $10^{-6} \mathrm{M}$ DES to the medium, the tyrosinase activity of melanocytes was significantly increased, with $\mathrm{P}<0.05$. In conclusion, a certain concentration of DES promoted the proliferation of melanocytes, enhanced the activity of tyrosinase and promoted pigment synthesis of melanocytes, with the optimal concentration of $10^{-6} \mathrm{M}$.
\end{abstract}

\section{Introduction}

Certain pigmented diseases, such as melasma, often occur in females. These hyperpigmentations result from an increase in melanin and its treatment remains a challenge. The epidermal pigmentation and skin malignant melanoma may be associated

Correspondence to: Professor Qin Li or Professor Biao Cheng, Department of Plastic Surgery, The Key Laboratory of Trauma Treatment and Tissue Repair of Tropical Area, People's Liberation Army, HuaBo BioPharmaceutical Institute of Guangzhou, General Hospital of Guangzhou Military Command, 111 Liuhua Road, Guangzhou, Guangdong 510010, P.R. China

E-mail: gzzxwk@163.com

E-mail: chengbiaocheng@163.com

Key words: diethylstilbestrol, melanocytes, cell proliferation, tyrosinase with estrogen. The pigmented spot becomes more severe and malignant melanoma proceeds rapidly during an abnormal menstruation or pregnancy. There are changes in the estrogen level in these physiological processes. Therefore, estrogen is considered an important factor that affects pigmented diseases (1). Diethylstilbestrol (DES) is a synthetic nonsteroidal estrogen that can produce the same pharmacological effects as natural estrogen (2). In order to explore the mechanism, the excess skin following eyelid blepharoplasty was collected for melanocyte culture and different DES concentrations were used to detect the effect on the proliferation and tyrosinase activity of melanocytes.

\section{Materials and methods}

Reagent. DES (D4628; Sigma, St. Louis, MO, USA), M254 melanocytes culture medium (M-254-500; Invitrogen Life Technologies, Carlsbad, CA, USA), Human Melanocytes Growth Supplement (HMGS, S-002-5; Invitrogen Life Technologies), $0.25 \%$ neutral proteinase (Beijing Solarbio Science \& Technology Co., Ltd., Beijing, China), 0.25\% trypsin mixed with $0.02 \%$ EDTA, newborn calf serum (Sijiqing Biotechnology Co., Hangzhou, China), 0.1\% L-DOPA (D9628; Sigma) and S-100 monoclonal antibody (BMO120; Wuhan Boster Biological Technology, Ltd., Wuhan, China) were used.

Drug preparation. An electronic balance was used to weigh $26.8 \mathrm{mg}$ DES. It was dissolved in $10 \mathrm{ml}$ dehydrated ethanol. A $50-\mu 1$ solution was removed and diluted with $5 \mathrm{ml} \mathrm{M} 254$ culture medium; $10^{-4} \mathrm{M}$ DES was acquired. Subsequently, it was diluted into $10^{-5}, 10^{-6}, 10^{-7}$ and $10^{-8} \mathrm{M}$ as final concentrations. The solutions were preserved at $4^{\circ} \mathrm{C}$ in darkness.

Melanocytes cultured. The excess skin following eyelid blepharoplasty was collected for cell culture. Informed consent was obtained from all the patients and it was approved by the Institutional Review Board. The specimen was soaked with povidone-iodine solution for $5 \mathrm{~min}$ and was subsequently flushed with saline. The subcutaneous tissue and part of the dermis was cut off. The specimen was cut into $0.5-\mathrm{mm}$ strips. The tissue was soaked in $0.25 \%$ neutral protease and preserved overnight at $4^{\circ} \mathrm{C}$. The epidermis was removed and digested by $0.25 \%$ trypsin and $0.02 \%$ EDTA at $37^{\circ} \mathrm{C}$ for $5 \mathrm{~min}$. The 
digestion was stopped by serum. A single-cell suspension could be acquired after being blown by pipette. Following this it was filtered by 200-mesh and centrifuged at $400 \mathrm{x}$ g for $16 \mathrm{~min}$, twice. The M254 medium mixed with HMGS was added to the cell suspension. The cell was seeded with a density of $5 \times 10^{5}$ in a $25-\mathrm{cm}^{2}$ culture bottle, which was incubated at $37^{\circ} \mathrm{C}$ and $5 \%$ $\mathrm{CO}_{2}$. The medium was changed after $48 \mathrm{~h}$. When melanocytes grew to $70-80 \%$ fusions, $0.25 \%$ trypsin mixed with $0.02 \%$ EDTA was added for digestion. The cells were gently blown, and serum was added to stop the digestion. Centrifugation was performed at $400 \mathrm{xg}$ for $6 \mathrm{~min}$, twice, and the supernatant was discarded. The fresh M254 medium and HMGS was added for passage, and cultured with a density of $5 \times 10^{5}$ cells in a $25-\mathrm{cm}^{2}$ culture bottle. The medium was changed firstly after $24 \mathrm{~h}$, and then every 3-4 days.

Melanocyte identification. The second generation of cells were seeded on a slide, and subsequently fixed by cold acetone. L-DOPA $(0.1 \%)$ was added and incubated for $4 \mathrm{~h}$ at $37^{\circ} \mathrm{C}$ for DOPA staining. Both DOPA and immunohistochemical staining of S-100 were used for identification.

Effect of different concentrations of exogenous DES on melanocyte proliferation by the MTT method. The third-generation melanocytes in the logarithmic growth phase were used. Following trypsin digestion, the cell concentration was adjusted to $2.5 \times 10^{4} / \mathrm{ml}$ and inoculated in a 96-well culture plate. There were 6 groups in total; 3 wells for each group and $200 \mu \mathrm{l}$ of cell suspension for each well. After incubation for $24 \mathrm{~h}$, the medium was changed and different DES concentrations in the medium were added to wells in different plates, with blank medium and medium containing $10^{-8}, 10^{-7}, 10^{-6}$, $10^{-5}$ and $10^{-4} \mathrm{M}$ DES. Each group was cultured for 5 days, and subsequently $20 \mu \mathrm{l}$ MTT $(5 \mathrm{mg} / \mathrm{ml})$ was added to each well. The cells were incubated for $4 \mathrm{~h}$, and the supernatant was discarded. To each well $150 \mu$ l dimethylsulfoxide was added. The cells were oscillated for $10 \mathrm{~min}$, and the absorbance value [optical density (OD)] was measured with the enzyme mark instrument (Bio-Cell Ltd., Ramat Gan, Israel), with an excitation wavelength of $490 \mathrm{~nm}$.

Effects of DES on melanocyte tyrosinase activity by the L-DOPA reaction. When the melanocytes had reached $70-80 \%$ confluence, the cells were digested and seeded according to the method for the MTT assay. A total of 7 groups (group 1 contained medium with no cells, group 2 contained no DES and groups 3-7 contained medium with $10^{-8}, 10^{-7}, 10^{-6}, 10^{-5}$ or $10^{-4} \mathrm{M}$ DES), with 3 wells for each group. The melanocytes were inoculated for $72 \mathrm{~h}$. Subsequently, the supernatant was discarded and the cells were washed with phosphate-buffered saline twice. Triton X-100 (100 $\mu \mathrm{l} ; 10 \mathrm{ml} / \mathrm{l})$ was added to each well and the cells were agitated for $5 \mathrm{~min}$, before $100 \mu \mathrm{l}$ L-DOPA (0.1\%) was added and incubated for $2 \mathrm{~h}$ at $37^{\circ} \mathrm{C}$. The absorbance value A was measured by the enzyme mark instrument with $490 \mathrm{~nm}$ wavelength. The activity of tyrosinase $=\left(\mathrm{OD}_{\text {group X }}-\mathrm{OD}_{\text {group 1 }}\right) /\left(\mathrm{OD}_{\text {group } 2}-\mathrm{OD}_{\text {group } 1}\right) \times 100 \%$, (group X refers to groups 3-7).

Statistical analysis. Results are expressed as mean \pm standard deviation. Statistical analysis was performed with the
SPSS 13.0 (SPSS, Inc., Chicago, IL, USA) software. All the data were analyzed by analysis of variance followed by the post hoc Bonferroni/Dunnet multiple comparison test. $\mathrm{P}<0.05$ was considered to indicate a statistically significant difference.

\section{Results}

Melanocytes culture. Primary culture of cells from the skin samples began to adhere after $24 \mathrm{~h}$, with some multi-dendritic cells scattered among the epidermal cells under inverted microscopy. The multidendritic cells had $\geq 2$ dendrites and an orbicular-ovate nucleus. Epidermal cells did not grow well and appeared to be slightly adherent in the melanocyte culture medium, which was discarded following medium exchange (Figs. 1 and 2).

Cell identification. Following DOPA staining, black particles could be observed in the cytoplasm and dendrites after light microscopy analysis (Fig. 3). Anti-S-100 protein immunohistochemistry showed yellow staining in the cytoplasm and dendrites, which was strong positive (Fig. 4).

MTT assay. The OD value changes reflect the effect of different concentrations of exogenous estrogen DES on the proliferation of human melanocytes. The proliferation of cultured melanocytes in vitro was induced by $10^{-8}-10^{-6} \mathrm{M}$ DES. The intensity was positively correlated with the drug concentration. Concentrations of $>10^{-5} \mathrm{M}$ DES presented inhibition or toxicity effects on the melanocytes growth (Figs. 5 and 6). The variance analysis showed that $10^{-8}, 10^{-7}$ and $10^{-6} \mathrm{M}$ concentration groups compared with the control groups had significant differences $(\mathrm{P}<0.05)$ (Fig. 7).

$L-D O P A$ reaction for tyrosinase activity. As shown in Fig. 8, different concentrations of exogenous estrogen DES had different results for the tyrosinase activity of melanocytes. The tyrosinase activity was significantly induced by $10^{-8}-10^{-6} \mathrm{M}$ DES, compared with the control group $(\mathrm{P}<0.05)$. The maximum effect was for $10^{-6} \mathrm{M}$ DES incubation, whereas $10^{-4} \mathrm{M}$ DES inhibited the tyrosinase activity.

\section{Discussion}

In humans, estrogen may be involved in numerous physiological processes of skin and hair follicles, such as skin aging, hair loss (3), wound healing and scar formation (4). Hyperpigmentation has been documented during pregnancy (melasma), in women ingesting oral contraceptives containing estrogens and in female and male infants treated with ointments containing estrogens (5). Particular regions of the body appear to be affected, including the genitals, abdomen, face and mammary areola. These clinical observations suggest that melanocytes can respond to estrogens by increasing their levels of pigmentation (6). The association between the changes of estrogen and morbidity of melasma has been the focus of previous studies (7).

DES is a synthetic nonsteroidal estrogen, which can produce the same pharmacological effects as natural estrogen (2). Using physiological concentrations of DES, the effects of different concentrations of exogenous DES on cultured melanocyte 


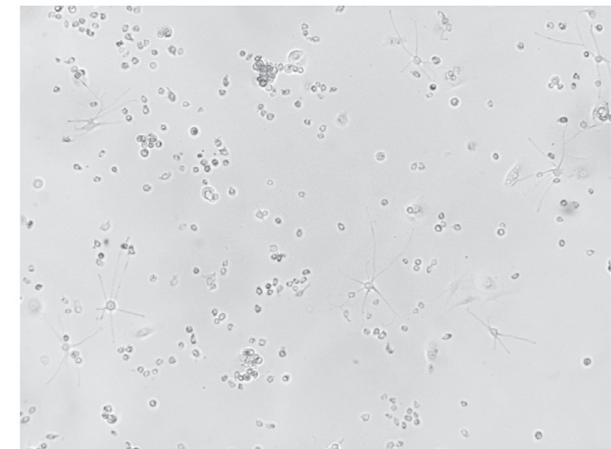

Figure 1. Primary culture of skin melanocytes. The gradual exfoliation of the epidermal cells (magnification, x200).

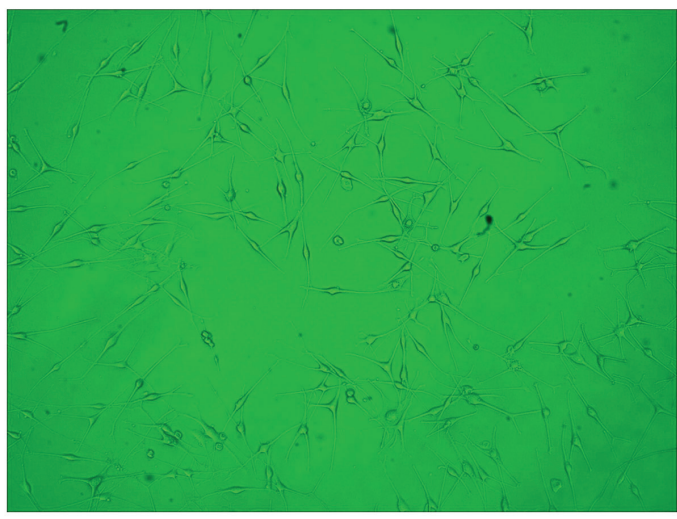

Figure 2. Melanocytes grew well after culture for 10 days (magnification, x200).

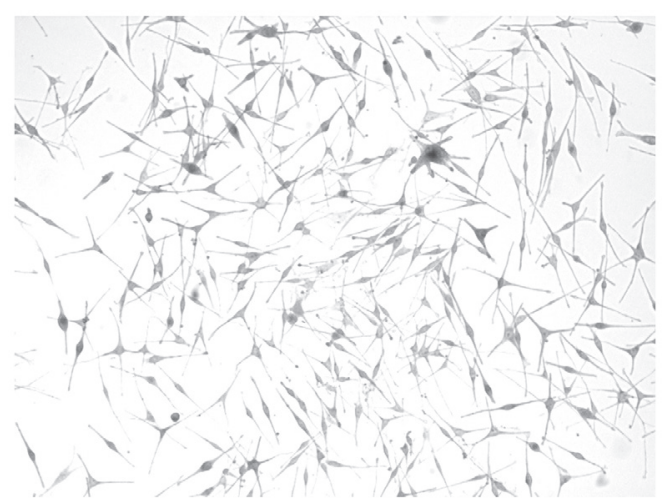

Figure 3. L-DOPA staining showing cytoplasmic staining [black particles (magnification, x200)].

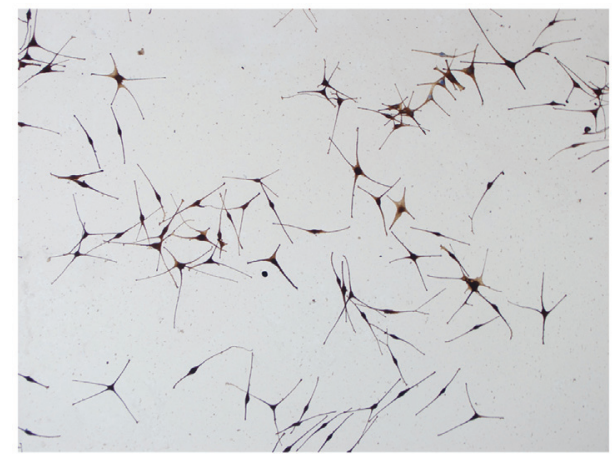

Figure 4. S-100 positive staining in the cytoplasm (magnification, $\mathrm{x} 200$ ).

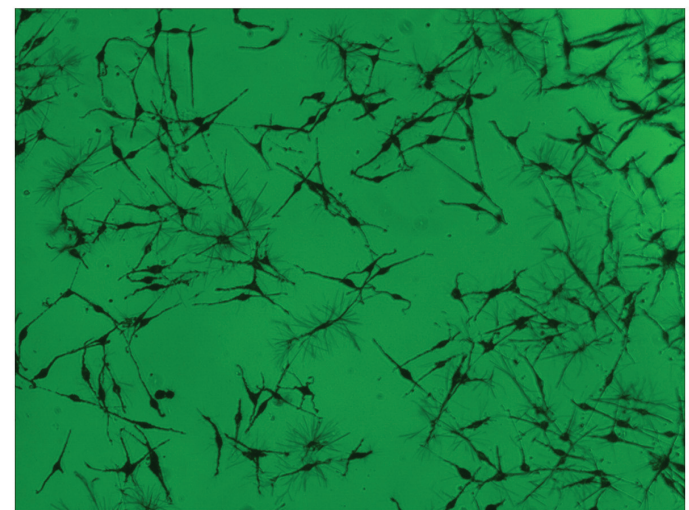

Figure 5. MTT staining when cultured in $10^{-7} \mathrm{M}$ diethylstilbestrol (magnification, $\mathrm{x} 200$ ).

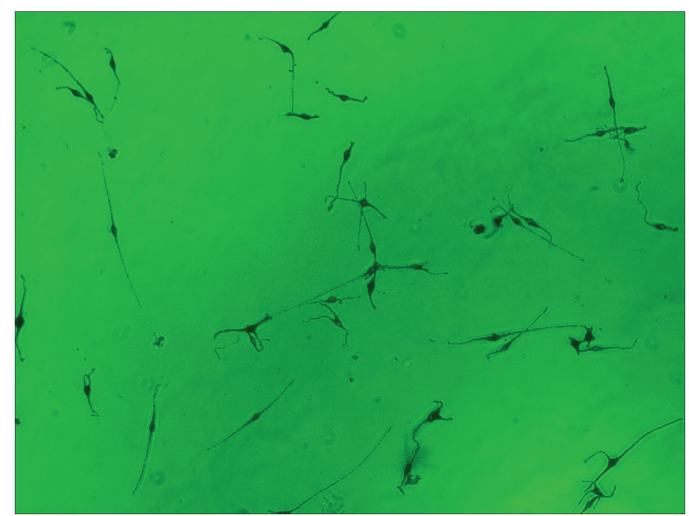

Figure 6. MTT staining when cultured in $10^{-5} \mathrm{M}$ diethylstilbestrol (magnification, $\mathrm{x} 200)$.

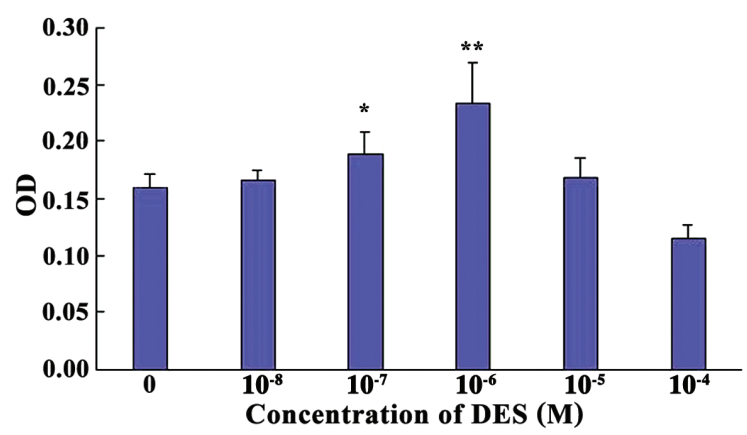

Figure 7. Effect of different diethylstilbestrol (DES) concentrations on human melanocytes proliferation. OD, optical density. ${ }^{*} \mathrm{P}<0.05$ and ${ }^{* *} \mathrm{P}<0.01$.

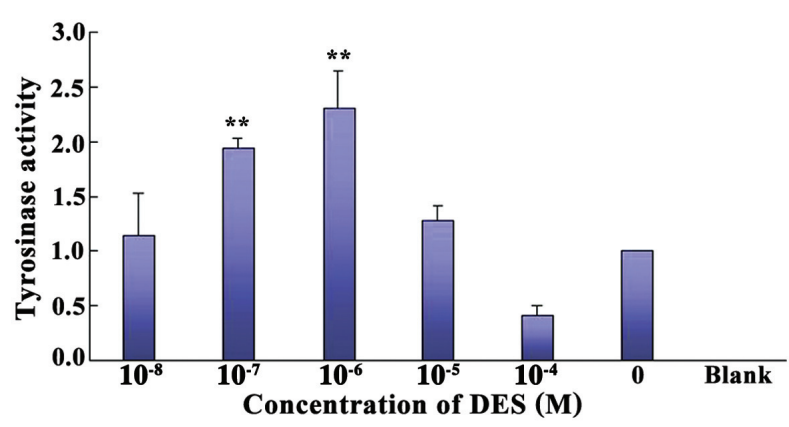

Figure 8. Effect of different diethylstilbestrol (DES) concentrations on the tyrosinase activity of melanocytes." $\mathrm{P}<0.01$. 
proliferation were assessed using the MTT assay and L-DOPA reaction. The results showed that the $10^{-8}-10^{-6} \mathrm{M}$ concentrations of DES promoted the proliferation of cultured melanocytes, and the intensity of the effect was positively correlated with the drug concentration. Up to $10^{-5} \mathrm{M}$ DES, the dendritics of cells were less, the shape became rounded, the cells grew slowly and the expansion ability decreased, which indicated that $>10^{-5} \mathrm{M} \mathrm{DES}$ produced inhibitory or toxic effects on the growth of melanocytes and inhibited the tyrosinase activity. The results suggest that by estrogen adjustment, the occurring estrogen-related pigmentation disorders may be reduced or improved.

Estrogen usually plays a role in combination with the estrogen receptor (ER). Despite strong clinical indications of an association between pregnancy and melanocytic responsiveness, no studies have successfully established a linkage between hormone responsiveness and nevi during pregnancy. The absence of ER $\alpha$ in melanocytes and melanoma discouraged further investigation. A second form of the receptor, $\mathrm{ER} \beta$, was also discovered (8). ER $\alpha$ is mainly expressed in the sebaceous gland cells, while it is weakly expressed in the epidermis. ER $\beta$ has a stronger expression in keratinocytes, including the epidermal basal layer and prickle cell layer $(9,10)$.

Estrogens can signal in a multifaceted manner involving diverse receptors that modulate genomic or non-genomic pathways, which in turn may have independent, synergistic or opposing actions. In addition, cell specific co-factors are also required and ligands that exhibit estrogenic activity in certain cells, paradoxically exhibit estrogen antagonism in others. Other studies have shown that DES could promote the generation of pro-opiomelanocortin and $\alpha$ melanocyte-stimulating hormone in melanocytes $(11,12)$, which promotes the proliferation of melanocytes and pigment synthesis.

Estrogen deficiency following menopause results in atrophic skin changes and acceleration of skin aging. Estrogen administration has positive effects on human skin by delaying or preventing skin aging manifestations. However, the use of estrogen replacement is a risk factor in breast and uterine cancer (13). Through the present experiment, we further verified the role of estrogen in the human skin pigment formation. How to establish the balance of estrogen to prevent estrogen-related skin aging and pigmentation disorder disease is a challenge.

\section{Acknowledgements}

The present study was supported by the CMA-L'oreal China skin grant 2010 (no. S2010070816), the National Basic Science and Development Programme of China (973 Programme, grant. no. 2012CB518105), the Natural Science Foundation of Guangdong Province of China (grant no. 06019692) and the Guangzhou Health Collaborative Innovation of Science and Technology Plan Projects of major projects (grant no. 201508020253).

\section{References}

1. Thornton MJ: Estrogens and aging skin. Dermatoendocrinol 5: 264-270, 2013.

2. Thornton MJ: Oestrogen functions in skin and skin appendages. Expert Opin Ther Targets 9: 617-629, 2005.

3. Zouboulis CC, Chen WC, Thornton MJ, Qin K and Rosenfield R: Sexual hormones in human skin. Horm Metab Res 39: 85-95, 2007.

4. Lieberman R and Moy L: Estrogen receptor expression in melasma: Results from facial skin of affected patients. J Drugs Dermatol 7: 463-465, 2008.

5. Stevenson S and Thornton J: Effect of estrogens on skin aging and the potential role of SERMs. Clin Interv Aging 2: 283-297, 2007.

6. Rowas SA, Haddad R, Gawri R, Al Ma'awi AA, Chalifour LE, Antoniou J and Mwale F: Effect of in utero exposure to diethylstilbestrol on lumbar and femoral bone, articular cartilage, and the intervertebral disc in male and female adult mice progeny with and without swimming exercise. Arthritis Res Ther 14: R17, 2012.

7. Kim NH, Cheong KA, Lee TR and Lee AY: PDZK1 upregulation in estrogen-related hyperpigmentation in melasma. J Invest Dermatol 132: 2622-2631, 2012.

8. El Safoury O, Rashid L and Ibrahim M: A study of androgen and estrogen receptors alpha, beta in skin tags. Indian J Dermatol 55: 20-24, 2010.

9. Ohata $\mathrm{C}$, Tadokoro $\mathrm{T}$ and Itami S: Expression of estrogen receptor beta in normal skin, melanocytic nevi and malignant melanomas. J Dermatol 35: 215-221, 2008.

10. Pelletier G and Ren L: Localization of sex steroid receptors in human skin. Histol Histopathol 19: 629-636, 2004

11. Rousseau K, Kauser S, Pritchard LE, Warhurst A, Oliver RL, Slominski A, Wei ET, Thody AJ, Tobin DJ and White A: Proopiomelanocortin (POMC), the ACTH/melanocortin precursor, is secreted by human epidermal keratinocytes and melanocytes and stimulates melanogenesis. FASEB J 21: 1844-1856, 2007.

12. Hiramoto $\mathrm{K}$, Kobayashi $\mathrm{H}$, Ishii $\mathrm{M}$, Sato $\mathrm{E}$ and Inoue $\mathrm{M}$ : Increased alpha-melanocyte-stimulating hormone (alpha-MSH) levels and melanocortin receptors expression associated with pigmentation in an $\mathrm{NC} / \mathrm{Nga}$ mouse model of atopic dermatitis. Exp Dermatol 19: 132-136, 2010.

13. Indran IR, Zhang SJ, Zhang ZW, Sun F, Gong Y, Wang X, Li J, Erdelmeier CA, Koch E and Yong EL: Selective estrogen receptor modulator effects of epimedium extracts on breast cancer and uterine growth in nude mice. Planta Med 80: 22-28, 2014. 